\title{
Frequency of Anxiety in Patients With Drug Poisoning in Rafsanjan City, Iran, in 2013
}

\author{
Fereshteh Solhdoost, ${ }^{1}$ Rezvan Sadr Mohammadi, ${ }^{2}$ Amir Moghadam Ahmadi, ${ }^{3}$ Reza Bidaki, \\ Seyed Ali Mostafavi, ${ }^{5}$ Mojde Bahmanyar, ${ }^{6}$ Sahar Rezaei Nejad, ${ }^{6}$ and Mohamad Hossein \\ Ahmadie $^{7}$ \\ ${ }_{2}^{1}$ Department of Psychiatry, Islamic Azad University, Yazd Branch, Yazd, IR Iran \\ ${ }_{2}^{2}$ Department of Clinical Psychology, Kar Higher Education Institute of Rafsanjan, Rafsanjan, IR Iran \\ ${ }^{3}$ Department of Neurology, Rafsanjan University of Medical Sciences, Rafsanjan, IR Iran \\ 4 Department of Psychiatry, Research Center of Addiction and Behavioral Sciences, Shahid Sadoughi University of Medical Sciences, Yazd, IR Iran \\ 5 Psychiatry Research Center, Roozbeh Hospital, Tehran University of Medical Sciences, Tehran, IR Iran \\ 6 Rafsanjan University of Medical Sciences, Rafsanjan, IR Iran \\ $7_{\text {Islamic Azad University, Yazd Branch, Yazd, IR Iran }}$ \\ ${ }^{*}$ Corresponding author: Reza Bidaki, Department of Psychiatry, Research Center of Addiction and Behavioral Sciences, Shahid Sadoughi University of Medical Sciences, Yazd, IR Iran. \\ Tel: +98-3536232003, Fax: +98-3532633555, E-mail: Reza_Bidaki@yahoo.com
}

Received 2014 June 16; Revised 2015 January 12; Accepted 2015 February 10.

\begin{abstract}
Background: Expansion of technology and progression of sciences have led to wider access to agricultural and industrial drugs and chemicals, which has resulted in many problems. Both in wanted and unwanted ways people may take these toxic agents, which perhaps may be along with many unpredictable, life-threatening and mortal outcomes. Based on many studies, most of intentional poisonings arise from an origin of patients' psychological backgrounds, which confirms necessity and importance of the recent study.

Objectives: This study aimed to study the prevalence of psychiatric disorders in patients with drug poisoning.

Patients and Methods: In this descriptive cross-sectional study, 116 cases of poisoning referred to emergency room of Ali-Ebn-Abitaleb Hospital in Rafsanjan city, Iran, were randomly selected. Frequency of anxiety among participants was evaluated through the Spielberger questionnaire. A past history of depression and obsession was also evaluated through interview by a psychiatrist based on diagnostic and statistical manual of mental disorders (DSM-5) criteria. All data were then collected and analyzed using SPSS version 17.

Results: Anxiety, as the second most common reason of psychiatric disorders in cases of poisoning, with a prevalence of $12.1 \%$ in form of mild, $75.9 \%$ moderate and $12.1 \%$ severe, was confirmed among the participants of this study.

Conclusions: The prevalence of anxiety among cases of poisoning with both drugs and chemicals are noticeably higher than general population. This fact strongly suggests the necessity of in-advance consultation and treatment of any underlying psychiatric disorders of patients to prevent prospective complications.
\end{abstract}

Keywords: Poisoning, Drug Side Effects, Anxiety

\section{Background}

Poisoning constitute a branch of medical emergency. Sometimes a few minutes delay in treatment may have serious consequence for patient, thus to save the lives of patients immediate and urgent measures will be required (1). Acute pesticide poisoning is an important cause of morbidity and mortality in Iran and worldwide (2). The general patterns of poisoning show a lot of variations among the developing countries $(3,4)$. The complex multifactorial etiology of suicide suggests the need to consider gender differences when developing effective strategies for suicide prevention (5). In Asia, There were $46 \%$ male and $54 \%$ female to attempt suicide with the age range 24.97 years, and the largest occupational groups were self-employed then house-keeping and low socio economic status. The most common group of educational level was high school and then middle school. The most commonly agents misused for suicide attempts were medicines and then pesticides. The most common drug groups were benzodiazepines and then antidepressant and antiepileptic medications (1).

Of 26078 emergency patients, 11465 were admitted for acute poisoning. Among them, 45 patients who came to the emergency room were died. The death was happened in $1 \%$ cases that the most common causes were opioids, medicines and pesticide poisoning. Twenty out of 50 studied patients were males and 30 were females. The most common age group that has been reported was 15 - 24 and then 25 - 45 years old. Seventy eight percent

Copyright (C) 2015, Zahedan University of Medical Sciences. This is an open-access article distributed under the terms of the Creative Commons Attribution-NonCommercial 4.0 International License (http://creativecommons.org/licenses/by-nc/4.0/) which permits copy and redistribute the material just in noncommercial usages, provided the original work is properly cited. 
(78.3\%) attempted suicide and the most common complications were respiratory and heart problems (6). A review of U.S. poison center data for 2004 showed over 12,000 exposures to tricyclic antidepressants (TCAs) (7), and over 40,000 exposures to salicylate-containing products (8).

A study by Jesus Alberdi et al. (9) in psychiatric center of La Courna University in Spain was performed in 2011 and described the effective factors on recurrent attempted suicide during 10 years on 5424 patients. Of 5424 patients, 4509 cases had attempted suicide again, and the results showed that a previous suicide is the main risk factor for repeating suicide, also other risk factors include: age, gender, low living status, job, and the previous psychiatric diagnosis. Of psychiatric problems affective disorders followed by personality disorder and schizophrenia were the priorities. The comparative study of suicide in people with or without mental disorder was conducted on 392 patients that had suicide attempt from October 2005 until June 2008. The findings show a significant difference in selection of the suicide method in patients with mental disorders compared to those without mental disorders (9). The experience of previous studies in recent decades emphasize on the important role of psychiatric problems in suicide attempts including self-induced poisoning. In our country, the exact statistics of the toxicity and its complications are not available. Therefore, to control poisoning we need to know the causes of this phenomenon.

\section{Objectives}

The aim of this study was to survey the prevalence of psychiatric disorders in patients with drug poisoning.

\section{Patients and Methods}

This is a descriptive and cross-sectional study.

\subsection{The Characteristics of the Study Population}

This study was conducted on patients with poisoning admitted to the emergency section of Ali-Ebn-Abitaleb Hospital in Rafsanjan city, Iran.

Inclusion criteria included people who have been poisoned.

Exclusion criteria included 1) people who have suffered a loss of consciousness and have been admitted to the intensive care unit (ICU), 2) People who have overdosed medications for any reasons, for example, a person with dementia who had consumed too much medicine. A sample size of 116 patients was randomly selected and studied for drug poisoning.

\subsection{Instruments and Procedures}

All patients who were referred due to poisoning were studied by filling out the questionnaires and entering into an interview by a psychiatrist. They filled out a demographic questionnaire which was a researcher-made questionnaire to assess demographic characteristics.

Past history of depression and obsessive compulsive disorder (OCD) was also evaluated through face-to-face interview of a psychiatrist with patients based on the diagnostic and statistical manual of mental disorders, fifth edition(DSM-5) criteria. Also, patients' data for anxiety were collected through state trait anxiety inventory (STAI) (Spielberger, 1983). The STAI is a widely used 40item measure of enduring (trait) and transient (state) anxiety symptoms, with well-supported reliability and validity. The state anxiety score was used in the analyses and focuses on how you are feeling in this moment. Reported scores ranged from 0 to 86 (10). Zamani et al. (11) have tested the validity of this questionnaire using the factor analysis method. Factor analysis showed that this test consists of three main structures as state anxiety, lack of anxiety and trait anxiety, which is a good indicator of reliability. The three factors describe $49 \%$ of variances. Test-retest reliability showed coefficients between $0.73-0.86$. Also, it had good correlation $(r=0.75-0.85)$ with other anxiety scales such as the Taylor anxiety scale, which indicates a good criterion validity of the instrument. Zamani et al. showed that Cronbach's alpha coefficient for trait anxiety in male subjects was 0.79 and in females was 0.84 and for state anxiety Cronbach's alpha coefficient was 0.85 in males and 0.89 in females (11).

\subsection{Data Collection and Analysis}

Data obtained from interviews and questionnaires were collected and then analyzed using SSPS version 17 software (SPSS Inc. Released 2008. SPSS Statistics for Windows, Version 17.0. Chicago, USA). The chi- square test was used to analyze the data, and information was provided by frequency, mean and standard deviation.

\section{Results}

This study included 116 poisoned patients referred to Ali-Ebn-Abitaleb emergency section in Rafsanjan city, with mean age of $24.6 \pm 7$ years and the range was $14-49$ years. The cases of the study included 71 male (61\%) and 45 females (39\%). Participants were divided into two age groups: 14 - 24 years (65 patients; 56\%) and 25 - 49 years (51 patients; $44 \%$ ).

Of all participants, 78 cases (67.5\%) were single, and 34 (29.5\%) were married, and 4 (3\%) were widowed or divorced. Of these cases 79 patients $(68 \%)$ were living in urban and 37 (32\%) were living in rural areas.

In terms of the education level, 34 patients (29.3\%) had college education, 34 (29.3\%) had high school education, $32(27.5 \%)$ had guidance school education and $16(13 \%)$ had primary school education. In regard to the occupation of patients, 36 cases (31\%) were employed, 35 (30\%) were student and collegian, 24 (20\%) were housekeeper, 12 (10\%) were unemployed, and 9 cases ( $7 \%$ ) were worker.

Among medications and poisons, $19.8 \%$ of poisoned patients consumed analgesics, 19\% consumed benzodiaze- 
Solhdoost F et al.

pine, $15.5 \%$ opium, $14.7 \%$ organophosphorous, $9.5 \%$ TCA, $9.5 \%$ alcohol, 3.4 rat poison, 5.6\% pesticide, $0.9 \%$ tacked barbiturates, $0.9 \%$ amphetamines, and $1.7 \%$ consumed detergent.

In terms of relative frequency of anxiety, 88 cases $(75.9 \%)$ were moderate, 14 (12.1\%) were mild and 14 (12.1\%) were affected with severe type. Table 1 shows the frequency of different levels of anxiety in subjects according to gender.

In this study, a mild anxiety score was $32-42$, the moderate 43 - 53 and the severe was 54 or above. According to the above results moderate anxiety in males has the highest value (80.3\%), but severe anxiety in them is the lowest level (7\%). The relationship between gender and anxiety was tested using the chi-square test after classification and disapproved with non-significant $P$ value $(P=0.113)$. So, we cannot infer that anxiety was different in both sexes.

Table 2 shows frequency of patients with different levels of anxiety after classification for age. According to the results shown in table 2 , in the age group of 14 - 24 years, moderate type of anxiety (80\%) has the highest rate and mild anxiety (9.2\%) was the lowest level. There was no significant relationship between anxiety and age groups $(\mathrm{P}=0.466)$. Therefore, severity of anxiety in both groups is alike.

Table 3 shows frequency of patients with different levels of anxiety after classification for marital status. According to Table 3, moderate type of anxiety (78.2\%) was the highest level in single persons and mild anxiety (0.0\%) was the lowest level in widowed or divorced. Results of the chi-square test showed no significant relationship between anxiety and marital status after classification among the participants $(\mathrm{P}=0.600)$. So, the severity of the anxiety does not depend on marital status.

Table 4 shows the frequency of patients with different levels of anxiety after classification for type of poisoning. Results of this study showed that the severe anxiety type (75\%) had the highest rate in cases of intentional poisoning, and mild anxiety $(9.7 \%)$ had the lowest rate of intentional poisoning. The results of the chi-square test showed no significant relationship between anxiety and type of poisoning in the participants $(\mathrm{P}=0.498)$.

Table 5 shows the frequency of patients with different levels of anxiety after classification for history of mental disorders. According to the table results, moderate type of anxiety (76.7\%), had the highest rate in individuals with a history of mental disorders and severe anxiety (10\%) had the lowest rate in individuals with a history of mental disorders. These relationships were tested by the chi- square test and were not significant $(\mathrm{P}=0.905)$.

There was no significant relationship between anxiety and a history of suicide attempt among the participants $(P$ value $=0.120)$.

Also, the severity of obsession in individuals with or without history of suicide was similar. Severity of obsession was significantly correlated with the level of education ( $\mathrm{P}=0.001)$. The highest severity of obsessions was seen in high school students. Severity of obsession was significantly correlated with social class $(\mathrm{P}=0.001)$. Obsession was so severe in the lower social classes.
Table 1. The Frequency of Patients with Different Levels of Anxiety According to Gender ${ }^{\mathrm{a}}$

\begin{tabular}{lccc}
\hline Gender & Mild & Moderate & Severe \\
\hline Male & $9(12.7)$ & $57(80.3)$ & $5(7)$ \\
Female & $5(11.1)$ & $31(68.9)$ & $9(20)$ \\
Total & $14(12.1)$ & $88(75.9)$ & $14(12.1)$ \\
\hline
\end{tabular}

$\mathrm{a}_{\text {Values are presented as No. (\%). }}$

Table 2. The Frequency of Patients with Different Levels of Anxiety After Classification for Age ${ }^{\mathrm{a}}$

\begin{tabular}{lccc}
\hline Age & Mild & Moderate & Severe \\
\hline $\mathbf{1 4 - 2 4}$ & $6(9.2)$ & $52(80)$ & $7(10.8)$ \\
$\mathbf{2 5 - 4 9}$ & $8(15.7)$ & $36(70.6)$ & $7(13.7)$ \\
Total & $14(12.1)$ & $88(75.9)$ & $14(12.1)$ \\
\hline
\end{tabular}

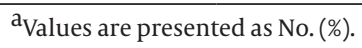

Table 3. The Frequency of Patients with Different Levels of Anxiety After Classification for Marital Status ${ }^{\text {a }}$

\begin{tabular}{lccc}
\hline Marital Status & Mild & Moderate & Severe \\
\hline Single & $10(12.8)$ & $61(78.2)$ & $7(9)$ \\
Married & $4(11.8)$ & $24(70.6)$ & $6(17.6)$ \\
Divorced & $0.0(0.0)$ & $3(75)$ & $1(25)$ \\
Total & $14(12.1)$ & $88(75.9)$ & $14(12.1)$ \\
\hline
\end{tabular}

${ }^{a}$ Values are presented as No. (\%).

Table 4. Frequency of Patients with Different Levels of Anxiety After Classification for Type of Poisoning ${ }^{a}$

\begin{tabular}{lccc}
\hline Type of Poisoning & Mild & Moderate & Severe \\
\hline Intentional & $7(9.7)$ & $11(15.3)$ & $54(75)$ \\
Unintentional & $17(38.6)$ & $18(40.9)$ & $9(20.5)$ \\
\hline
\end{tabular}

${ }^{a}$ Values are presented as No. (\%).

Table 5. The Frequency of Patients with Different Levels of Anxiety After Classification for History of Mental Disorders ${ }^{\mathrm{a}}$

\begin{tabular}{lccc}
\hline History of Mental Disorders & Mild & Moderate & Severe \\
\hline Yes & $4(13.3)$ & $23(76.7)$ & $3(10)$ \\
No & $10(11.6)$ & $65(75.6)$ & $11(12.8)$ \\
Total & $14(12.1)$ & $88(75.9)$ & $14(12.1)$ \\
\hline
\end{tabular}

${ }^{\mathrm{a}}$ Values are presented as No. (\%).

Severity of obsession was significantly associated with age. As with increasing age, severity of disorders is more severe $(\mathrm{P}=0.023)$. There was no significant association between the severity of obsession and sex, occupation, type of poisoning, residence, history of past suicide attempts 
and type of drug. The relative frequency of occurrence of OCD: the type of medium was max (38.8\%) and the lowest to severe OCD (31.9\%) and mild was $29.3 \%$.

The relationship between depression and history of mental disorders in the past $(\mathrm{P}=0.001)$, level of education $(\mathrm{P}=0.045)$ and the history of suicide attempts $(\mathrm{P}=$ 0.001 ) was significant. There was no significant relationship between the severity of depression and age, sex, occupation, type of poisoning, residence, and the type of suicide.

\section{Discussion}

This study was performed on 116 patients with poisoning. Sixty-one percent of the participants were males and $39 \%$ were females, which indicate the relative frequency of intoxication among men. A study by Ashkani et al. on 100 patients with poisoning included $46 \%$ male and $54 \%$ female at the Shiraz University of Medical Sciences in 2002 (1). Internationally, women are more likely than men to attempt suicide by deliberate self-poisoning, which may be due to increased vulnerability and susceptibility of women (12). However, the results of the present study showed that male participants with poisoning were more than females. This may have happened by chance and/or as a result of a limited sample size.

The mean age in our study was $24.6 \pm 7$ years. In studies that were performed by Kalkan et al. at Turkey (mean age of $34.7 \pm 1.3$ yaers) (13), and by Rafighdoost at Birjand University of Medical Sciences (an age range $=15$ to 24 ) (6), age group of youth had the most common prevalence of poisoning that has the same result with our studies.

According to the results of Carlsten et al. (12) the prevalence of poisoning in single people is significantly more than married ones. The result of this study is similar to ours. One reason for this report may be that those people who lived alone and those who have failed in love, are at higher risk (12). Based on studies conducted in India and England, the poisoning in single individuals is more than married, of course, it was greater in men (14). Marital problems, family conflict and less social support systems on the rights of women against men in family life may be the causes of higher toxicity in married women than men in external review (1).

In our study, the highest exposure was in high school and then college and guidance school.

In Ashkani study on 100 poisoned patients at the Shiraz University of Medical Sciences in 2002, similarly poisoning in education levels of high school and middle school were the most common (1). Our study and above analysis showed that increasing education level to high school rises the risk of suicide poisoning, which can be alarming for us.

A study conducted by Ashkani et al. at Shiraz University of Medical Sciences in 2002 showed that the most common occupation group which was susceptible was those with self-employment (1). We also reached the similar results. Can be said poisoning in the self-employment group can be triggered by status of the job and also access to toxic substances and among the educated class due to emotional problems, schools, family and social problems.

In a study by Rafighdoost that was performed on organophosphate poisoning on 50 patients in 2007 in Birjand city, Iran, $78.3 \%$ of cases have been intentional poisoning (6). Based on our results and previous reports, self-poisoning accounted for the highest percentage of toxicity. The high rate of intentional poisoning in society can be alarming merely requiring investigation.

In our study, the highest rate of consumption of medications was analgesics and then benzodiazepines and opiates. In Ashkani study, the most common drug group was benzodiazepines and then antidepressants (1).

From view of socioeconomic status: $39 \%$ with high social class, $35.5 \%$ with low social class and $29 \%$ were moderate. In a study by Eizadi et al. in Isfahan University in 2012, which was performed on 384 patients, most patients were in the high socioeconomic (15). In a study by Jesus Alberdi et al. in the psychiatric center of Lacoruna University in 2011 that was performed on 5824 patients, history of previous mental disorders, low social and economic status, and age are reported among the risk factors for deliberate poisoning (9). In Ashkani study, also one of the effective risk factors for deliberate poisoning was low socioeconomic status (1). Among the mentioned studies, only the former was in accordance with our statistical work and 2 others were opposed. The low socioeconomic status can be considered as one of the causes of voluntary poisoning, this is because of poverty and lack of essential facilities to have a good life. Nowadays, because of industrial life particularly in high social class and being away from each other, the deliberate poisoning rate in high socioeconomic status is growing up.

In our study, history of previous mental disorders was detected in $25 \%$ of the cases and $75 \%$ not listed. The results of a study by the Eizadi et al. in Isfahan on 385 patients showed that about $82.5 \%$ had a history of mental problems (15). A study conducted at the University of Pennsylvania in 2007 on 275 poisoning cases showed that the most causes of repeated suicide attempts were the prior psychiatric disorders. Jesus Alberdi showed that the effective risk factors on suicide attempt are prior psychiatric disorders. All of the above were opposed to our results and some reasons for this were: 1) Patients did not accept their mental disorders, 2) They did not trust to their therapists (9).

In our study, past history of suicide attempt in $81 \%$ of the participants was not reported. Only $19 \%$ of the participants reported a history of suicide. Also, according to the text book of psychiatry, the most common cause of suicide was a previous suicide attempt. All of the above were opposed to our findings and the reasons of this are: 1) poisoned patients who had a history of suicide 
in several times were referred to the psychiatric center, however, they refused to go to the psychiatric center because they are not honest about their suicide, 2) due to the small city and the fear of stigma for future career, education and marriage, they try to hide it. Since an unsuccessful suicide attempt may be followed by a successful suicide and eventually may lead to death, these people will have to pay more attention (14). Findings of the first study in regards with history of suicide attempts were similar to ours and the last two were not consistent with them. This could be the reason for need to studies with larger sample sizes and the study of other mental disorders that we have not dealt with. In our study one of the leading causes of deliberate self-poisoning is the severe depression that with an in time psychiatric treatment can be reduced. So, in those who had a history of psychiatric disorders in the past, severity of depression was higher. Causes of it can be the acceptance of patients from their self-psychological problems, being unable to perform their usual tasks and the rejection from their family. Also, the severity of depression in high school students is higher than other age groups because of their emotional and education problems (especially unsuccessful university entrance exam, push to go to military service etc.).

There was no significant relationship between the severity of anxiety and age, sex, socio-economic situation, occupation, type of poisoning, residence, history of suicide in the past and the type of suicide. The results of the above-mentioned studies were in consistent with the findings of our study.

There was a significant relationship between the severity of depression and past history of mental disorders ( $P$ $=0.045)$. Depressive symptoms are frequent among suicide attempters. However, a subgroup of male attempters reporting interpersonal conflicts is characterized by a lower level of depression (16).

History of mental disorders in the past was significantly correlated with OCD. Severity of obsession was significantly correlated with the level of education. There was no significant association between the severity of obsession and sex, occupation, type of poisoning, residence, history of past suicide attempts and type of drug. Obsession alone is not a risk factor for the action of intentional poisoning. If illness, particularly depression and drug abuse be accompanied by obsession, action of poisoning or suicide attempt happens more.

Prevalence of anxiety in poisoning caused by a variety of toxins and medications are common in the general population; so, this justifies the need for counseling and treatment of these disorders before the event.

\section{Acknowledgments}

The authors would like to thank the personnel of AliEbn-Abitaleb Hospital in Rafsanjan city, who helped us in this research and the authors would also like to thank the members of the research committee of the Ali-EbnAbitaleb faculty of Islamic Azad University in Yazd City, Iran. This article is extracted from a medical student's thesis to obtain the doctoral degree in medicine at Islamic Azad University of Yazd which has been registered with number 912004.

\section{Footnote}

Authors' Contribution:Fereshteh Solhdoost helped us in doing the study; Rezvan Sadr Mohammadi, and SeyedAli Mostafavi wrote and revised the study; Sahar Rezaei Nejad and Mojde Bahmanyar wrote the study; Amir Moghadam Ahmadi: design and concept of the study; Mohammad Hossein Ahmadieh: data collection and analysis, and Reza Bidaki participated in all stages of work.

\section{References}

1. Ashkani H, Touhidi M, Moeeni SA. A study of suicidal attempts by drugs and poisonous substances in emergency rooms and intensive care units of hospitals affiliated with Shiraz University of Medical Sciences. J Medic res. 2002;1(1):48.

2. Soltaninejad K, Faryadi M, Sardari F. Acute pesticide poisoning related deaths in Tehran during the period 2003-2004. J Forensic Leg Med. 2007;14(6):352-4. doi: 10.1016/j.jflm.2006.12.011. [PubMed: 17631456]

3. Tagwireyi D, Ball DE, Nhachi CF. Poisoning in Zimbabwe: a survey of eight major referral hospitals. JAppl Toxicol.2002;22(2):99-105. [PubMed: 11920933]

4. Yang CC, Wu JF, Ong HC, Hung SC, Kuo YP, Sa CH, et al. Taiwan National Poison Center: epidemiologic data 1985-1993. J Toxicol Clin Toxicol.1996;34(6):651-63. [PubMed: 8941193]

5. Dedic G. Gender differences in suicide in Serbia within the period 2006-2010. Vojnosanit Pregl. 2014;71(3):265-70. [PubMed:24697013]

6. Rafighdoost A. Epidemiological study of respiratory and cardiac effects of organophosphate poisoning. J Birjand Univ Med Sci. 13(4):949-53.

7. Woolf AD, Erdman AR, Nelson LS, Caravati EM, Cobaugh DJ, Booze LL, et al. Tricyclic antidepressant poisoning: an evidence-based consensus guideline for out-of-hospital management. Clin Toxicol (Phila). 2007;45(3):203-33. doi: 10.1080/15563650701226192. [PubMed: 17453872]

8. Chyka PA, Erdman AR, Christianson G, Wax PM, Booze LL, Manoguerra AS, et al. Salicylate poisoning: an evidence-based consensus guideline for out-of-hospital management. Clin Toxicol (Phila). 2007;45(2):95-131. doi: 10.1080/15563650600907140. [PubMed: 17364628]

9. Alberdi-Sudupe J, Pita-Fernandez S, Gomez-Pardinas SM, IglesiasGil-de-Bernabe F, Garcia-Fernandez J, Martinez-Sande G, et al. Suicide attempts and related factors in patients admitted to a general hospital: a ten-year cross-sectional study (1997-2007). BMC Psychiatry. 2011;11:51. doi: 10.1186/1471-244x-11-51. [PubMed: 21453478]

10. Spielberger CD. Manual for the State Trait Anxiety Inventory (STAI). Palo Alto, CA: Consulting Psychologists Press; 1983.

11. Zamani-Asl Z. The preliminary validation of State -Trait Anxiety Inventory in Tehran' educational and guidance. Tehran: University of Tehran;1991.

12. Carlsten A, Waern M, Allebeck P. Suicides by drug poisoning among the elderly in Sweden 1969-1996. Soc Psychiatry Psychiatr Epidemiol.1999;34(11):609-14. [PubMed:10651180]

13. Kalkan S, Cevik AA, Cavdar C, Aygoren O, Akgun A, Ergun N, et al. Acute methanol poisonings reported to the Drug and Poison Information Center in Izmir, Turkey. Vet Hum Toxicol. 2003;45(6):334-7. [PubMed: 14640491]

14. Werth JL. Rational suicide and the role of values. Focus. 2000;15(5):5-6. [PubMed: 12180384] 


\section{Solhdoost F et al.}

15. Eizadi-Mood N, Saghaei M, Alfred S, Zargarzadeh AH, Huynh C, Gheshlaghi F, et al. Comparative evaluation of Glasgow Coma Score and gag reflex in predicting aspiration pneumonitis in acute poisoning. J Crit Care. 2009;24(3):470.e9-15. doi: 10.1016/j. jcrc.2008.08.008. [PubMed: 19327309]
16. Toth MD, Adam S, Birkas E, Szekely A, Stauder A, Purebl G. Gender differences in deliberate self-poisoning in Hungary: analyzing the effect of precipitating factors and their relation to depression. Crisis. 2014;35(3):145-53. doi: 10.1027/0227-5910/a000245. [PubMed: 24491825] 